\title{
Error Bounds for the Gauss-Chebyshev Quadrature Formula of the Closed Type
}

\author{
By M. M. Chawla
}

1. Introduction. We are concerned with the Gauss-Chebyshev quadrature formula of the closed type,

$$
\int_{-1}^{1}\left(1-t^{2}\right)^{-1 / 2} f(t) d t=\sum_{k=0}^{n} A_{k} f\left(t_{k}\right)+E_{n}(f) \quad(n \geqq 2)
$$

with the abscissas

$$
t_{k}=\cos (k \pi / n), \quad k=0, \cdots, n,
$$

and the Christoffel numbers

$$
A_{0}=A_{n}=\pi / 2 n, \quad A_{k}=\pi / n, \quad k=1, \cdots, n-1 .
$$

The quadrature formula ( 1 ) is exact for all polynomials of degree $\leqq 2 n-1$. For a general discussion of the Gauss formulas of the closed type, see Krylov [1, Chapter 9].

The usual real-variable theory estimate for the error $E_{n}(f)$ is given (Krylov $[1$, p. 171]) in terms of derivatives of $f$ :

$$
E_{n}(f)=-\frac{\pi}{2^{2 n-1}} \frac{f^{(2 n)}(n)}{(2 n) !}
$$

for some $\eta \in[-1,1]$. The error expression (2) is valid for the class of functions which are $2 n$-times differentiable. In most cases, the exact value of $\eta$ will be unknown, and the estimate $\max _{-1 \leqq t \leqq 1}\left|f^{(2 n)}(t)\right|$ is used. But in many cases it will be far from convenient to obtain $f^{(2 n)}$ or the bounds on it.

In the following, we use the complex-variable method to obtain a contour integral representation for $E_{n}(f)$, applied to analytic functions, and give bounds for the error in terms of the size of the integrand in the complex plane.

2. Error Bounds. Let $f(t)$ be analytic on $[-1,1]$, then it can be continued analytically so as to be single-valued and analytic in a domain $D$ of the $z$-plane containing the interval $[-1,1]$ in its interior.

Let $C$ be a closed contour in $D$ enclosing the interval $[-1,1]$ in its interior and let $U_{n-1}=2^{n-1} \prod_{k=1}^{n-1}\left(t-t_{k}\right)$ be the Chebyshev polynomial of the second kind. On applying the residue theorem to the contour integral

$$
\frac{1}{2 \pi i} \int_{C} \frac{f(z) d z}{w(z)}, \quad w(t)=\left(t^{2}-1\right) U_{n-1}(t),
$$

we get

Received February 13, 1967. 


$$
f(t)=\sum_{k=0}^{n} \frac{w(t)}{\left(t-t_{k}\right) w^{\prime}\left(t_{k}\right)} f\left(t_{k}\right)+\frac{1}{2 \pi i} \int_{C} \frac{w(t) f(z)}{w(z)} d z .
$$

Multiplying (4) by the weight $\left(1-t^{2}\right)^{-1 / 2}$ and integrating on $[-1,1]$, there results the quadrature formula (1), with the error

$$
E_{n}(f)=\frac{i}{\pi} \int_{C} \frac{Q_{n-1}^{*}(z) f(z) d z}{U_{n-1}(z)\left(z^{2}-1\right)}
$$

where we have put

$$
Q_{n}{ }^{*}(z)=\frac{1}{2} \int_{-1}^{1}\left(1-t^{2}\right)^{1 / 2} \frac{U_{n}(t)}{z-t} d t .
$$

In a recent paper (Chawla [2]), the following result was proved. For sufficiently large $|z|$,

$$
\left|\frac{Q_{n}^{*}(z)}{U_{n}(z)}\right| \leqq \frac{\pi}{2^{2 n+2}}|z|^{-2 n-1} .
$$

Taking $C:|z|=R$ with sufficiently large $R$, from (5) and (7), we find

$$
\left|E_{n}(f)\right| \leqq \frac{\pi}{2^{2 n-1}} \frac{R^{2} M(R)}{\left(R^{2}-1\right) R^{2 n}},
$$

where $M(R)=\left.\left.\max \right|_{z}\right|_{=R}|f(z)|$.

These error bounds are simple to obtain and they will not be unduly pessimistic, but are valid for the class of functions which are continuable analytically in a sufficiently large domain of the $z$-plane containing the range of integration $[-1,1]$.

We obtain next estimates for $E_{n}(f)$ for all functions analytic on [-1, 1]. For this purpose, we introduce the ellipse $\varepsilon_{\rho}(\rho>1)$ defined by

$$
z=\frac{1}{2}\left(\xi+\xi^{-1}\right), \quad \xi=\rho e^{i \theta} \quad(0 \leqq \theta \leqq 2 \pi)
$$

with foci at $z= \pm 1$ and semiaxes $\frac{1}{2}\left(\rho+\rho^{-1}\right)$ and $\frac{1}{2}\left(\rho-\rho^{-1}\right)$.

Let $f(t)$ be analytic on $[-1,1]$. Then, for some $\rho>1, f$ can be continued analytically into the closure of an ellipse $\varepsilon_{\rho}$. It has been proved (Chawla [2]) that for $z$ on $\varepsilon_{\rho}$,

$$
Q_{n}^{*}(z)=(\pi / 2) \xi^{-n-1}
$$

Since on $\varepsilon_{\rho}$,

$$
U_{n}(z)=\left(\xi^{n+1}-\xi^{-n-1}\right) /\left(\xi-\xi^{-1}\right)
$$

and by virtue of (10), (5) becomes

$$
E_{n}(f)=i \int_{|\xi|=\rho} \frac{f\left[\frac{1}{2}\left(\xi+\xi^{-1}\right)\right] d \xi}{\xi\left(\xi^{2 n}-1\right)}
$$

or, equivalently,

$$
E_{n}(f)=i \int_{\varepsilon_{\rho}} \frac{f(z) d z}{\left(z^{2}-1\right)^{1 / 2}\left[\left(z \pm\left(z^{2}-1\right)^{1 / 2}\right)^{2 n}-1\right]},
$$


where the sign in the integrand is chosen so that $\left|z \pm\left(z^{2}-1\right)^{1 / 2}\right|>1$. From (12) follows the following estimate for the error:

$$
\left|E_{n}(f)\right| \leqq 2 \pi M(\rho) /\left(\rho^{2 n}-1\right)
$$

where $M(\rho)=\max |f|$ on $|\xi|=\rho$.

By experimenting with various "admissible" $\rho$, a conservative upper bound can be established. A similar remark applies to the estimate (8).

3. Example. Consider the estimation of error in the evaluation of the integral

$$
J=\int_{-1}^{1}\left(1-t^{2}\right)^{-1 / 2} \frac{a t}{4+t}=\frac{\pi}{(15)^{1 / 2}} \doteq 0.811155735192
$$

by the quadrature formula (1). For $n=4$, the approximate value found by the quadrature formula is $\doteq 0.811155845096$. Thus, the true error $E_{4} \doteq-0.0000001099$.

In this case, the real-variable estimate (2) gives $\left|E_{4}\right| \leqq 0.0000012$.

Taking $R=3.5$, the estimate (8) gives $\left|E_{4}\right| \leqq 0.0000023$.

However, evaluating the contour integral in (13), we find

$$
E_{4}=-\frac{2 \pi}{(15)^{1 / 2}\left[\left(4+(15)^{1 / 2}\right)^{8}-1\right]} \doteq-0.0000001099
$$

which is the exact error.

Department of Mathematics Indian Institute of Technology Hauz Khas, New Delhi-29

India \#2008.

1. V. I. KryLov, Approximate Calculation of Integrals, Macmillan, New York, 1962. MR 26

2. M. M. Chawla, "On the Chebyshev polynomials of the second kind," SIAM Rev., v. 9, 1967 , p. 729 . 